\title{
Growth of Supermassive Black Holes, Galaxy Mergers and Supermassive Binary Black Holes
}

\author{
S. Komossa ${ }^{1}$, J. G. Baker ${ }^{2}$ and F. K. Liu ${ }^{3}$ \\ ${ }^{1}$ Max-Planck-Institut für Radioastronomie, Auf dem Hügel 69, 53121 Bonn, Germany \\ email: skomossa@mpifr.de \\ ${ }^{2}$ NASA/GSFC, Mail Code: 663, Greenbelt, MD 20771 \\ ${ }^{3}$ Department of Astronomy, Peking University, Beijing 100871, China
}

\begin{abstract}
The study of galaxy mergers and supermassive binary black holes (SMBBHs) is central to our understanding of the galaxy and black hole assembly and (co-)evolution at the epoch of structure formation and throughout cosmic history. Galaxy mergers are the sites of major accretion episodes, they power quasars, grow supermassive black holes (SMBHs), and drive SMBH-host scaling relations. The coalescing SMBBHs at their centers are the loudest sources of gravitational waves (GWs) in the Universe, and the subsequent GW recoil has a variety of potential astrophysical implications which are still under exploration. Future GW astronomy will open a completely new window on structure formation and galaxy mergers, including the direct detection of coalescing SMBBHs, high-precision measurements of their masses and spins, and constraints on $\mathrm{BH}$ formation and evolution in the high-redshift Universe.
\end{abstract}

Keywords. galaxies, black holes, galaxy mergers, gravitational waves

\section{Introduction}

Electromagnetic observations have provided us with numerous insights into the cosmic growth history of black holes and the (co-)evolution with their host galaxies. Important advances of recent years include the discovery of quasars beyond redshift $z=6.5$, population studies in deep and wide field surveys constraining the luminosity function and $\mathrm{BH}$ mass function out to high redshift, detailed studies of nearby galaxy mergers, and the emergence of binary active galactic nuclei (AGN), candidate SMBBHs, and candidate recoiling SMBHs. These have been accompanied by substantial progress on the theory side, including cosmological simulations of large-scale structure, galaxy merger simulations at super-high resolution and approaching the "final parsec", breakthroughs in numerical relativity enabling simulations of SMBBH coalescences, and intense ongoing investigations of potential electromagnetic signals quasi-simultaneous with binary coalescence.

Despite this progress, a number of important questions related to the growth and cosmological evolution of $\mathrm{BHs}$ remain partially unanswered:

(a) How and when did the first BHs form?

(b) How and when did they grow and evolve? $\dagger$

(c) How do they evolve with respect to their host galaxies?

(d) How often do binary SMBHs coalesce?

(e) What is the $\mathrm{BH}$ spin evolution?

$(f)$ How frequent is GW recoil, and what are its astrophysical implications?

$\dagger$ What is the relative contribution of gas accretion, BH-BH mergers, and stellar tidal capture/disruption? What are the timescales, rates, efficiencies, and trigger mechanisms? 
In the future, all of these questions can be independently addressed by GW observations from space and using pulsar timing arrays $(\S 6)$.

\section{Early SMBH growth and the highest-redshift quasars}

Particularly tight constraints on the early $\mathrm{BH}$ growth have emerged from the discovery of luminous high-redshift quasars. At the time of writing, seven were known beyond redshift $z>6.5$ (Mortlock et al. 2011; de Rosa et al. 2014; Venemans et al. 2015), including the one at highest redshift of $z=7.1$ (Mortlock et al. 2011), corresponding to a cosmic age of only 700 Myr.

Black hole masses of objects seen at those redshifts are high (Figure 1), on the order of $\sim 10^{9} \mathrm{M}_{\odot}$ and beyond, reaching $\sim 10^{10} \mathrm{M}_{\odot}$ in two cases (at $z=6.3$, Wu et al. 2015; and $z=5.4$, Wang et al. 2015), implying that very massive BHs were in place early in the Universe. They do not grow much beyond that anywhere in the cosmos (Figure 1) as mass estimates of the most massive BHs amount to a few $10^{10} \mathrm{M}_{\odot}$ (e.g., Shen et al. 2011, but see also Brockamp et al. 2015).

How fast can massive BHs grow early in the Universe? BHs accreting at the Eddington limit,

$$
L_{\mathrm{Edd}}=\frac{4 \pi G M m_{\mathrm{p}} c}{\sigma_{\mathrm{T}}} \simeq 10^{38} \mathrm{erg} \mathrm{s}^{-1}\left(\frac{M}{M_{\odot}}\right),
$$

with accretion luminosity $L=\eta \dot{M} c^{2}$, where $\eta$ is the radiative efficiency, grow exponentially, as

$$
M=M_{\text {init }} e^{t / \tau_{\text {salpeter }}} \text { with } \tau_{\text {salpeter }}=4.510^{7}(\eta / 0.1) \mathrm{yr} .
$$

The growth timescale depends on the radiative efficiency $\eta$ and therefore on BH spin. Higher BH spin implies last stable orbits closer in, higher radiative efficiency, and therefore less rapid BH growth. For instance, it takes $\sim 2$ Gyr for a BH to grow up to $10^{9} \mathrm{M}_{\odot}$ if it is rapidly spinning $(\eta=0.3)$. Even if BHs start accreting with low spin, the accretion process itself (if from a coherent, long-lived disk), will rapidly spin up the hole (Volonteri et al. 2013). The detection of high-redshift quasars with SMBH masses as high as $\sim 10^{10} M_{\odot}$ at $z=6.3$ (Wu et al. 2015) then implies that there is not enough time for them to grow via Eddington-limited accretion from low-mass seed BHs. Supercritical accretion and/or massive seeds are possible solutions (e.g., Volonteri et al. 2015).

With future GW observations $(\S 6)$ we will be sensitive not only to the most massive black holes, but also to high-redshift mergers of black holes as much as a million times smaller. These observations will motivate new questions not only about how the biggest black holes grew but about the yet unseen broader population of objects, including the precursors of the typical SMBHs observed in the present day Universe.

\section{Galaxy mergers}

Gas-rich galaxy mergers trigger quasar activity, provide the fuel source for BH growth, are the sites of feedback processes, and likely drive the BH-host scaling relations.

\subsection{Triggering of quasar activity}

The idea that luminous quasars are activated by galaxy interactions came up early, in the seventies (e.g., Stockton \& MacKenty 1983). Since then, numerous studies have been carried out to address whether all AGN activity is triggered by mergers. Based on a recent compilation of results from several IR, optical and X-ray surveys up to $z \sim 3$, Treister et al. (2012) concluded that the fraction of AGN in mergers is a strong function of, and 


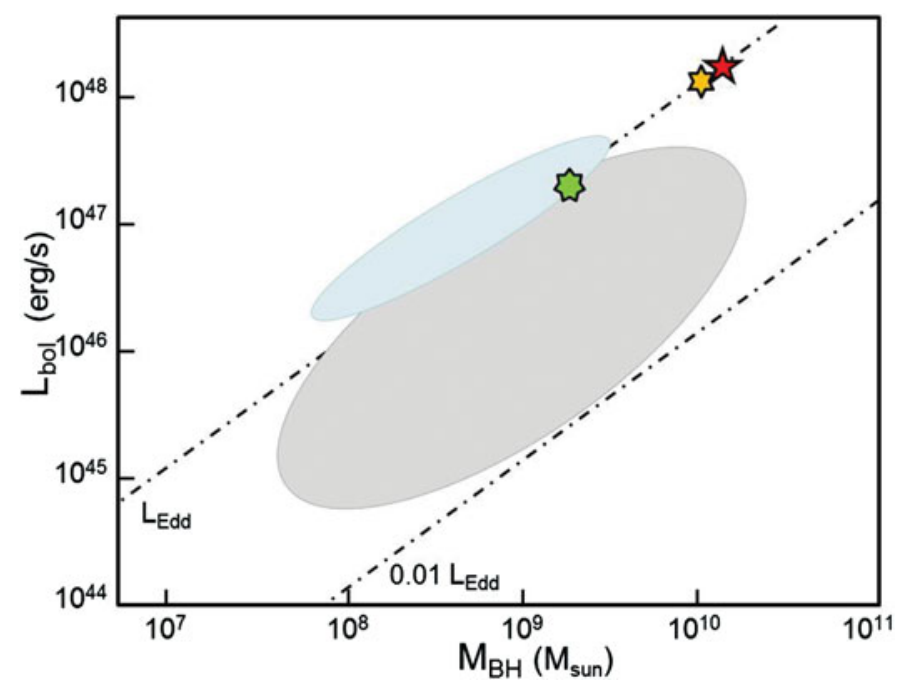

Figure 1. BH masses of high-redshift quasars (star symbols, from left to right: ULAS J1120+0641 at $z=7.1$, J0306+1853 at $z=5.4$, and SDSS J0100+2802 at $z=6.3$ ) in comparison with other $z>6$ quasars (small grey ellipse), and the area populated by a large number of lower-redshift SDSS quasars (large grey ellipse; adopted from Wang et al. 2015).

increases with, AGN luminosity. The majority of the most luminous quasars all reside in major mergers. $\dagger$ Lower-luminosity AGN, on the other hand, are likely triggered by secular processes like bar-driven inflows, stochastic cloud accretion events, minor mergers and perhaps stellar captures (Hopkins \& Hernquist 2009). These differences may also explain cosmic downsizing (Brandt \& Hasinger 2005). While the low-mass, low-luminosity AGN make the majority in number, a large part of the total $\mathrm{BH}$ mass growth likely occurrs in the most luminous quasars (e.g., Treister et al. 2012). Runaway BH growth in these gas-rich environments is plausibly prevented by feedback processes, which have also been invoked to explain the scaling relations between BHs and their host galaxies.

\subsection{BH-host scaling relations}

The mass of the central SMBH correlates tightly with a number of host galaxy properties including stellar velocity dispersion and total bulge mass $\ddagger$ (Graham 2015). Galaxy mergers are thought to be the main driver behind these relations, either by merging repeatedly with each other (Jahnke \& Macciò 2011), or by triggering feedback processes such that the radiation from the accretion disk heats the ambient gas and drives strong outflows until - at a critical BH mass - the remaining gas is expelled which then terminates further BH growth and fixes the host properties (e.g., Hopkins et al. 2006).

\section{Evolution of galaxy mergers and SMBBHs}

Galaxies merge frequently throughout cosmic times. Whenever both galaxies harbor SMBHs at their centers, the formation of a binary SMBH is inevitable. The merger evolves in several stages (Begelman et al. 1980; our Figure 2). (1) The early stages of galaxy merging are driven by dynamical friction. (2) At close separations, on the order of parsecs, the two SMBHs form a bound pair. The further shrinkage of their orbit then

$\dagger$ Not all samples show this trend (e.g., Villforth et al. 2014)

$\ddagger$ A few extreme outliers of very massive BHs in low-mass host galaxies have been reported recently (e.g., van den Bosch et al. 2012; Trakhtenbrot et al. 2015). 


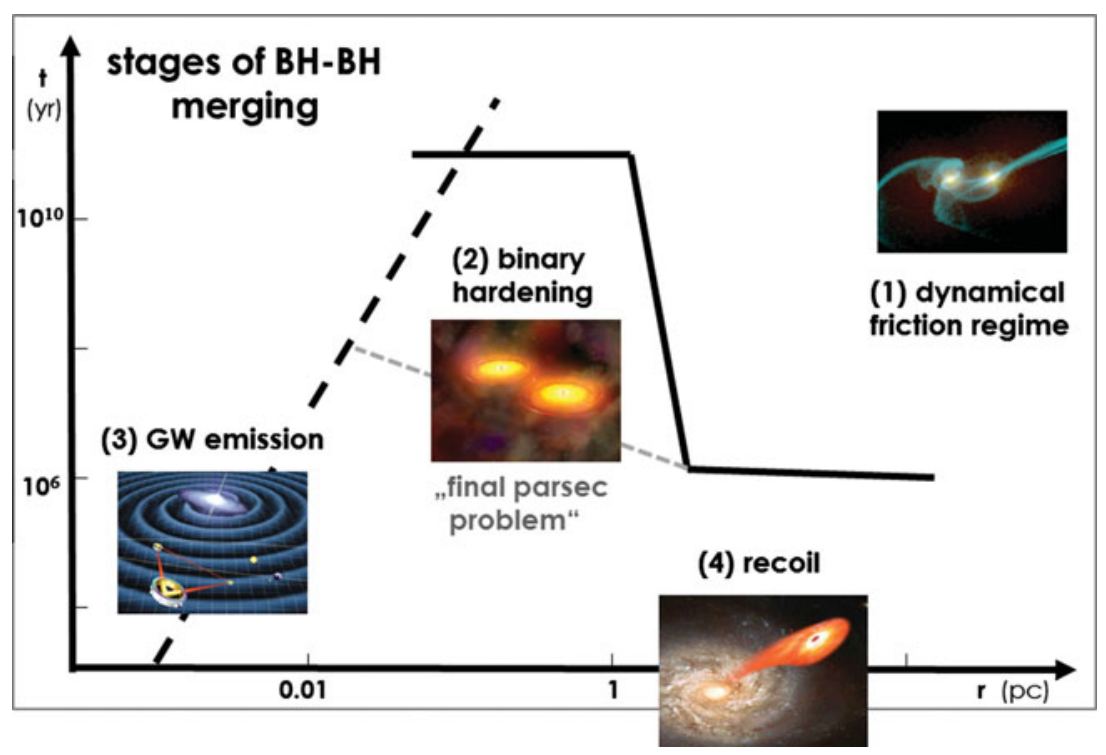

Figure 2. Stages of galaxy and SMBBH merging.

depends on the efficiency of interactions with gas and stars in carrying away energy and angular momentum; known as the "final parsec problem" (Colpi 2014). (3) At separations well below a parsec, emission of GWs becomes efficient leading to further orbital shrinkage and final coalescence. This GW-driven regime can be thought of as proceeding in three stages; the inspiral phase, dynamical merger, and final ringdown, which emit characteristic GW radiation (e.g., Hughes 2002; Centrella et al. 2010). (4) Depending on the orbital configuration including masses and spins of the binary, after coalescence the newly formed single SMBH then recoils with a velocity as high as $\sim 5000 \mathrm{~km} \mathrm{~s}^{-1}$ (Lousto \& Zlochower 2011; Sperhake 2015) but typically much lower.

\section{Observations of (U)LIRGs, binary AGN and SMBBHs}

Prime examples of gas-rich galaxy mergers are luminous and ultra-luminous infrared galaxies [(U)LIRGs, Sanders et al. 1988]. These allow us to study the stages of galaxy merging in the nearby Universe, and are more abundant in the higher-redshift Universe, where they become a major galaxy population. Based on an analysis of HST images, Kartaltepe et al. (2012) concluded, that at least $70 \%$ of ULIRGs at redshift $z \sim 2$ reside in interacting systems. Nearby (U)LIRGs provide us with excellent laboratories for studying the physics and evolution of galaxy mergers, and the triggering of AGN activity at their centers (e.g., Iwasawa et al. 2011). One of the first observed with highresolution X-ray imaging spectroscopy with Chandra was the nearby ULIRG NGC 6240, which let to the detection of a pair of X-ray luminous AGN at its center (Komossa et al. 2003).

Meanwhile, more cases of AGN pairs have emerged, based on X-ray, radio and optical imaging spectroscopy. The majority of these is found in galaxy pairs in relatively early stages of their evolution (Deane et al. 2014, Fig. 2, for a compilation of wide systems at $\leqslant 10 \mathrm{kpc}$ spatial separation; Comerford et al. 2015; Fu et al. 2015; De Rosa et al. 2015, and references therein). Only 3-5 systems are currently known which host binary AGN below $\sim 1 \mathrm{kpc}$ spatial separation, which are located in single galaxies or advanced mergers. Besides NGC 6240, these are J0402+379 in the radio (at only 7 pc projected separation, 
Rodriguez et al. 2006; Burke-Spolaor 2011), SDSS J1323-0159 in the optical (Woo et al. 2014), NGC 3393 in X-rays (Fabbiano et al. 2011; see Koss et al. 2015 for a different interpretation), and SDSS J1502+1115 (Deane et al. 2014; see Wrobel et al. 2014 for a different interpretation). All of these nearby pairs, upon their final coalescence, would be easily detectable in GWs with a LISA-type mission (e.g., Colpi 2014, Fig. 1). At present, radio-VLBI observations provide us with the most powerful technique of resolving SMBH pairs at small angular separation.

The most compact systems, supermassive binary BHs, can no longer be spatially resolved with current techniques, and we therefore rely on indirect methods when searching for them electromagnetically. Most search strategies are based on signs of semiperiodicity, for instance in lightcurvest or in the spatial structures of radio jets. Others include double-peaked emission lines or unusual spectral energy distributions (SEDs) at the time when the advanced binary has opened a gap in the inner accretion disk (see Komossa \& Zensus 2015 for a review of signatures and pre-2015 SMBBH candidates). Several recent candidates have emerged from large-sky surveys and long-term monitoring programs. PG 1302-102 (Graham et al. 2015; D'Orazio et al. 2015; Kun et al. 2015), PSO J334.2028+01.4075 (Liu et al. 2015), and PG 1553+113 (Ackermann et al. 2015) all show pronounced semi-periodic lightcurve variability; while Mrk 231 (Yan et al. 2015) exhibits a UV-dim SED characteristic of a binary-driven gap in the accretion disk.

All of these detection methods of SMBBHs require at least one $\mathrm{BH}$ to be active. In order to trace the population of inactive binaries, Liu et al. (2009) proposed to take advantage of epochs of temporary activity in form of accretion flares from tidally disrupted stars in these systems. As the second $\mathrm{BH}$ temporarily interrupts the stellar debris stream on the primary, it causes characteristic dips and recoveries in the tidal disruption lightcurve. This signature has been identified in the lightcurve of SDSS J120136.02+300305.5, which is consistent with the presence of a sub-milliparsec SMBBH (Liu et al. 2014).

Finally, post-coalescence candidates include radio galaxies with characteristic structures [double-doubles or X-shaped systems (e.g., Liu et al. 2003; Roberts et al. 2015), interpreted as evidence for the interruption and re-start of accretion activity during coalescence, and/or BH spin flips], and recoiling black holes of which a few candidates have been identified in recent years (Komossa 2012).

\section{What do GWs tell us about structure formation?}

All of the questions raised in $\S 1$ can efficiently be addressed with GW astronomy with space-based observatories and pulsar timing arrays (e.g., Hughes 2002; Menou 2003; Centrella 2003; Sesana 2013; McWilliams et al. 2014; Colpi 2014; Barausse et al. 2015), in a completely independent and sometimes unique way.

We do not yet have any direct observations of the first seed BHs. They may form as light seeds from the collapse of the first massive stars, or as massive seeds through direct collapse of large gas clouds. In the near future, we will not be able to observe this regime directly electromagnetically, except perhaps through high-redshift GRBs. In GWs, and with an $e L I S A$-type mission, low-mass BHs would be observable out to redshifts $z<15$ (Colpi 2014); in a crucial regime of galaxy assembly and seed BH formation and growth.

Further, GWs allow us to measure luminosity distances, and then serve as standard candles (standard sirens, Schutz 1986; Barausse et al. 2015) $\ddagger$.

$\dagger$ The blazar OJ 287 with its $~ 12$ yr optical periodicity is one of the best-studied cases (e.g., Valtonen et al. 2012)

$\ddagger$ If potential problems posed by weak lensing can be overcome. 
While current searches for tightly bound SMBBHs continue to be challenging, and we still lack "smoking gun" signatures for incontrovertible identifications of such binaries, GW observations can provide a strongly complementary view. Where gravity is strong enough to produce observable GWs, it is expected to dominate the process, leading to clear GW signatures with very straightforward physical interpretations. Further, if multimessenger observations are possible then a few GW observations may be leveraged to eventually hone our interpretation of more numerous electromagnetic binary candidates.

In particular, GWs from coalescing binaries enable high-precision measurements of $\mathrm{BH}$ masses and spins. Measurements of coalescence rates (including extreme mass-ratio inspirals) inform us about the merger history of galaxies and constrain the accretion history (from spin measurements). Pulsar timing arrays have just started to place constraints on galaxy merger history from limits on the stochastic GW background (e.g., Zhu et al. 2014; Arzoumanian et al. 2015; Lentati et al. 2015; Shannon et al. 2015). GW astronomy therefore provides us with unique tracers of black hole assembly and growth, including new constraints on the importance of accretion, merging and stellar captures in growing black holes, and on the BH spin history.

\section{References}

Ackermann, M., et al. 2015, ApJ, in press; arXiv:1509.02063

Arzoumanian, Z., et al. 2015, ApJ, in press; arXiv:1508.03024

Barausse, E., Bellovary, J., Berti, E., Holley-Bockelmann, K., Farris, B., Sathyaprakash, B., \& Sesana, A. 2015, Barausse, E., Bellovary, J., Berti, E., et al. 2015, J. Phys. Conf. Ser., 610, 012001

Brandt, W. N. \& Hasinger, G. 2005, ARA\& $A, 43,827$

Brockamp, M., Baumgardt, H., Britzen, S., \& Zensus, A. 2015, A\&AA, in press; arXiv:1509.04782

Begelman, M. C., Blandford, R. D., \& Rees, M. J. 1980, Nature, 287, 307

Burke-Spolaor, S. 2011, MNRAS, 410, 2113

Centrella, J. M. 2003, "What can we learn about cosmic structure from gravitational waves?" in The Emergence of Cosmic Structure: Thirteenth Astrophysics Conference, AIP Conf. Proc., vol. 666, eds. S. S. Holt \& C. S. Reynolds (AIP: Melville, NY) p. 337

Centrella, J., Baker, J. G., Kelly, B. J., \& van Meter, J. R. 2010, Rev. Mod. Phys., 82, 3069

Colpi, M. 2014, SSRv, 183, 189

Comerford, J. M., Pooley, D., Barrows, R. S., et al. 2015, ApJ, 806, 219

Deane, R. P., et al. 2014, Nature, 511, 57

De Rosa, G., et al. 2014, ApJ, 790, 145

De Rosa, A., et al. 2015, MNRAS, 453, 214

D'Orazio, D. J., Haiman, Z., \& Schiminovich, D. 2015, Nature, 525, 351

Fabbiano, G., Wang, J., Elvis, M., \& Risaliti, G. 2011, Nature, 477, 431

Fu, H., Myers, A. D., Djorgovski, S. G., et al. 2015, ApJ, 799, 72

Graham, A. W. 2015, "Galaxy Bulges and Their Massive Black Holes: A Review," in Galactic Bulges, eds. E. Laurikainen, R. F. Peletier, \& D. A. Gadotti (Springer: Berlin) in press; arXiv:1501.02937

Graham, M. J., et al. 2015, Nature, 518, 74

Hopkins, P. F., Hernquist, L., Cox, T. J., et al. 2006, ApJS, 163, 1

Hopkins, P. F. \& Hernquist, L. 2009, ApJ, 694, 599

Hughes, S. A. 2002, MNRAS, 331, 805

Iwasawa, K., et al. 2011, A\&A, 529, 106

Jahnke, K. \& Macciò, A. V. 2011, ApJ, 734, 92

Kartaltepe, J. S., et al. 2012, ApJ, 757, 23

Komossa, S. 2012, Advances in Astronomy, 2012, 364973

Komossa, S., Burwitz, V., \& Hasinger, G., et al. 2003, ApJ, 582, L15

Komossa, S. \& Zensus, J. A. 2015, "Compact Object Mergers: Observations of Supermassive 
Binary Black Holes and Stellar Tidal Disruption Events," in Star Clusters and Black Holes Across Cosmic Times, Proc. IAU Symp. 312, eds. Y. Meiron et al. (Cambridge Univ. Press: Cambridge) in press; arXiv:1502.05720

Koss, M. J., et al. 2015, ApJ, 807, 149

Kun, E., Frey, S., Gabányi, K. È., et al. 2015, MNRAS, 454, 1290

Lentati, L., et al. 2015, MNRAS, 453, 2576

Liu, F. K., Wu, X.-B., \& Cao, S. L. 2003, MNRAS, 340, 411

Liu, F. K., Li, S., \& Chen, X. 2009, ApJ, 706, L133

Liu, F. K., Li, S., \& Komossa, S. 2014, ApJ, 786, 103

Liu, T., et al. 2015, ApJ, 803, L16

Lousto, C. \& Zlochower, Y. 2011, Phys. Rev. Lett., 107, 231102

McWilliams, S. T., Ostriker, J. P., \& Pretorius, F. 2014, ApJ, 789, 156

Menou, K. 2003, Classical Quant. Grav., 20, 37

Mortlock, D. J., et al. 2011, Nature, 474, 619

Roberts, D. H., Saripalli, L., \& Subrahmanyan, R. 2015, ApJ, 810, L6

Rodriguez, C., Taylor, G. B., Zavala, R. T., et al. 2006, ApJ, 646, 49

Sanders, D. B., Soifer, B. T., Elias, J. H., et al. 1988, ApJ, 325, 74

Schutz, B. F. 1986, Nature, 323, 310

Sesana, A. 2013, MNRAS, 433, L1

Shannon, R. M. et al. 2015, Science, 349, 1522

Shen, Y., et al. 2011, ApJS, 194, 45

Sperhake, U. 2015, "Gravitational Recoil and Astrophysical Impact," in Gravitational Wave Astrophysics, Astrophys. Space Sci. Proc., vol. 40, ed. C. F. Sopuerta (Springer: New York) p. 185

Stockton, A. \& MacKenty, J. W. 1983, Nature, 305, 678

Trakhtenbrot, B., et al. 2015, Science, 349, 168

Treister, E., Schawinski, K., Urry, C. M., \& Simmons, B. D. 2012, ApJ, 758, L39

Valtonen, M., Ciprini, S., \& Lehto, H. J. 2012, MNRAS, 427, 77

van den Bosch, R. C. E., Gebhardt, K., Gültekin, K., et al. 2012, Nature, 491, 729

Venemans, B. P., et al. 2015, ApJ, 801, L11

Volonteri, M., Sikora, M., Lasota, J.-P., \& Merloni, A. 2013, ApJ, 775, 94

Volonteri, M., Silk, J., \& Dubus, G. 2015, ApJ, 804, 148

Villforth, C., et al. 2014, MNRAS, 439, 3342

Wang, F., et al. 2015, ApJ, 807, L9

Woo, J.-H., Cho, H., Husemann, B., et al. 2014, MNRAS, 437, 32

Wrobel, J. M., Walker, R. C., \& Fu, H. 2014, ApJ, 792, L8

Wu, X.-B., et al. 2015, Nature, 518, 512

Yan, C.-S., Lu, Y., Dai, X., \& Yu, Q. 2015, ApJ, 809, 117

Zhu, X.-J., Wen, L., Hobbs, G., et al. 2014, MNRAS, 444, 3709 